\title{
NUEVA ESPECIE DE PSEUDOSINELLA SCHÄFFER, 1897 (COLLEMBOLA, ENTOMOBRYIDAE) DE CUEVAS DE LA SIERRA DE CAMEROS EN LA RIOJA, ESPAÑA
}

\author{
E. Beruete * y R. Jordana *
}

\begin{abstract}
RESUMEN
Se describe una nueva especie de Pseudosinella, . riojana sp. n., de las cuevas de la Sierra de Cameros, en la Rioja. Se da la descripción completa de la especie y las diferencias que la separa de las especies cercanas a ella por su morfología: $2+2$ ojos, base del labium con $\mathbf{M}_{1} \mathbf{m}_{2} \mathbf{r e l}_{1} \mathbf{l}_{2}(\mathrm{M}=$ ciliada, $\mathrm{m}=$ lisa), fórmula de macrosedas dorsales $\mathbf{R 0 0 1 / 0 / 0 0 / 0 1 0 1 + 2}$; quetotaxia del segmento abdominal II: _aBq $\mathbf{a q}_{\mathbf{2}}$.
\end{abstract}

Palabras clave: Collembola, Entomobryidae, Pseudosinella, fauna cavernícola, Península Ibérica.

\section{ABSTRACT \\ New species of Pseudosinella Schäffer, 1897 (Collembola, Entomobryidae) from caves of Cameros mountains in Rioja, Spain}

A new species of Pseudosinella, P. riojana n. sp., is described from Cameros mountains. Species description and morphological differences with nearest species is given: 2 +2 eyes, labium basis with setae $\mathbf{M}_{1} \mathbf{m}_{2} \mathbf{r e l}_{1} \mathbf{l}_{2}(\mathbf{M}=$ ciliated, $\mathrm{m}=$ smooth $)$, dorsal macro-

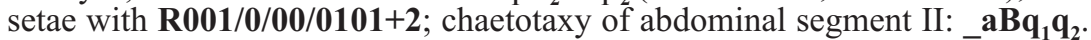

Key words: Collembola, Entomobryidae, Pseudosinella, cave fauna, Iberian Peninsula.

\section{Introducción}

Dentro de un estudio más amplio de la fauna de colémbolos del karst del norte de la Península Ibérica y del sudoeste de Francia, se ha estudiado la Cueva Lóbrega en la Sierra de Cameros, Rioja, y se han clasificado también los ejemplares recolectados por el Hogar Catalán de la Rioja (HCR) que amablemente nos ha cedido J. Armendáriz para su estudio. El esquema seguido en la descripción de esta especie ha sido el usual en el género. En la descripción realizada se ha añadido un dígito más a la fórmula de macrosedas dorsales con el fin de dar cabida en ella a la macroseda anterior al pseudopo- ro en el terguito torácico II, como describen Deharveng y Gouze (1986) en P. cabidochei y Deharveng (1988) en P. bessoni.

\section{Material y métodos}

Los ejemplares han sido recogidos manualmente en el interior de las cuevas, con un aspirador y fijados en alcohol al 70\%. Algunos de ellos se han montado en el líquido de Hoyer para su observación a microscopía óptica. Un paratipo ha sido deshidratado en la serie creciente de etanol hasta el $100 \%$, y luego ha sido sometido a su desecación a

* Departamento de Zoología y Ecología, Universidad de Navarra, Apartado 177, 31080, Pamplona. rjordana@unav.es 
punto crítico de $\mathrm{CO}_{2}$, en cámara de presión. Después fue montado en una platina (stub) de aluminio y sombreado con oro (16nm de deposición) en atmósfera de Argón con un K550 sputter coater de EMITECH. El ejemplar así montado ha sido observado con un Digital Scanning Electron Microscope DSM 940 A de ZEISS.

\section{Pseudosinella riojana sp. $\mathrm{n}$.}

Material eStudiado. Material tipo: Cueva Lóbrega (Torrecilla en Cameros, Rioja). SP1306. 880904. 31 ej. Leg. Beruete. Depositado en el Museo de Zoología de la Universidad de Navarra.

Holotipo: SP1306-02. Paratipos: SP1306-01. SP1306-03 (montado en platina para SEM) y 28 paratipos en alcohol.

Otro material: Peña Esquillas (Sta. Engracia de Jubera, Rioja). 821214. 2 ej. Sima del Maestro (Nestores, Rioja). 830515. 8 ej. Cueva del tejón (Ortigosa de Cameros, Rioja) 840218. 10 ej. Leg. J. Armendáriz (HCR, Hogar Catalán de la Rioja).

La localización de las cuevas con sus coordenadas UTM se da en la Tabla 1 y en la Figura 4.

DESCRIPCIÓN. Talla: 2.1-3.2 mm. El hábitus de la especie se muestra en la figura 1. En la cabeza están presentes dos manchas oculares a cada lado, pero en el estudio a microscopía electrónica de barrido estas manchas oculares se corresponden con dos corneolas algo levantadas de la cutícula, una de ellas de la mitad de diámetro a la otra, y con la cutícula con el mismo gránulo que el resto del cuerpo, lo que supone una regresión de los ojos en su adaptación a las cuevas. La cabeza, cuerpo y coxas con gránulos pigmentarios dispersos. La relación antena/diagonal cefálica oscila entre 2.2 y 2.5. La relación entre los artejos antenales I/II/III/IV es aproximadamente 0.18/0.38/0.47/0.61. Las largas antenas destacan por la gran cantidad y variedad de sedas. Se han contabilizado las siguientes: 1): Macro o microsedas normales, ciliadas, con una inserción característica de estas sedas formando un borde

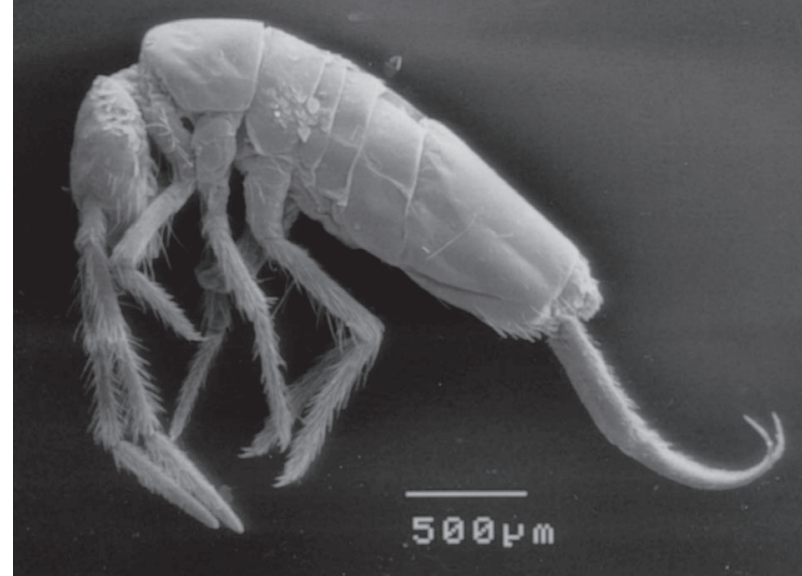

Fig. 1.- Hábitus de P. riojana sp. n.

Fig. 1.- Habitus of P. riojana n. sp.

quitinizado alrededor del alvéolo (Fig. 2, B1); estas sedas pueden variar entre 65 y 10 micras de longitud. (Fig. 2, A, 1, 2, 3, 4; Fig. 2, C1; Fig. 2, E1). 2): Sedas en las que el alvéolo está bordeado por gránulos. Dentro de este tipo de sedas se distinguen a su vez algunas formas. 2.1: Sedas finamente espinuladas desde la base (Fig. 2, E2) presentes en todos los artejos; 2.2: sedas más cortas, estriadas, finas (no llegan a una micra de diámetro) y agudas, que al microscopio óptico parecen lisas (Fig. 2, D1) y que son numerosas en la parte externa del artejo antenal IV y aparentemente son sedas s; 2.3: sedas s subcilíndricas, lisas, algo más gruesas, estriadas, de 5-6 micras de longitud, son menos numerosas y se encuentran en la parte dorso-externa del artejo antenal IV (Fig. 2, B2), y son semejantes a las sensilas de guarda del órgano sensorial del artejo antenal III; 2.4: sedas lisas, cortas que en su base presentan un doble toro esférico, como un doble anillo (Fig. 2, C2) y que están situadas en la parte interna del artejo antenal IV; 2.5: sedas largas, como las

Tabla 1.- Datos geográficos de localización de las cuevas donde se ha encontrado P. riojana sp. n.

Table 1.- Geografical data on caves localisation with P. riojana $\mathrm{n}$. sp.

\begin{tabular}{|c|c|c|c|c|c|c|c|c|}
\hline Nombre de la cueva & Provincia & Nº Catálogo & Huso & Zona & UTM & Altitud & Legit & Mapa \\
\hline Cueva Lóbrega (S. de Cameros, Torrecilla) & Rioja & LO ..../241 & $30 \mathrm{~T}$ & WM & ${ }^{53} 0100 / 4676700$ & 900 & Beruete & 1 \\
\hline Peña Esquillas (Sta. Engracia de Jubera) & Rioja & LO ..../242 & $30 \mathrm{~T}$ & WM & $557500 / 4685100$ & 630 & Armendáriz & 2 \\
\hline Maestro, Sima del (Nestores) & Rioja & LO ...././241 & $30 \mathrm{~T}$ & WM & $530500 / 4682100$ & 1220 & Armendáriz & 3 \\
\hline Tejón, C. del (Ortigosa de Cameros) & Rioja & LO ..../241 & $30 \mathrm{~T}$ & WM & $521700 / 4670600$ & 1400 & Armendáriz & 4 \\
\hline
\end{tabular}

Mapa: situación de las cuevas en la fig. 4 
Fig. 2.- Sedas de las antenas de $P$. riojana $\mathrm{sp}$. n. A: Artejo antenal I donde se señalan diferentes tipos de sedas. Barra $=20 \mu \mathrm{m}$. B: 1 , alvéolo de una macroseda y 2 , seda sensorial $\mathbf{s}$. Barra $=$ $2 \mu \mathrm{m} . \mathrm{C}: 1$, mesoseda normal; 2 , seda sensorial tipo 2.4. Barra $=2 \mu \mathrm{m}$. D: seda sensorial fina, larga y puntiaguda tipo 2.2. $\mathbf{s}$. Barra $=$ $2 \mu \mathrm{m}$. E: 1, mesosedas normales; 2, seda sensorial espinulada tipo 2.1. Barra= $2 \mu \mathrm{m}$. F: seda sensorial

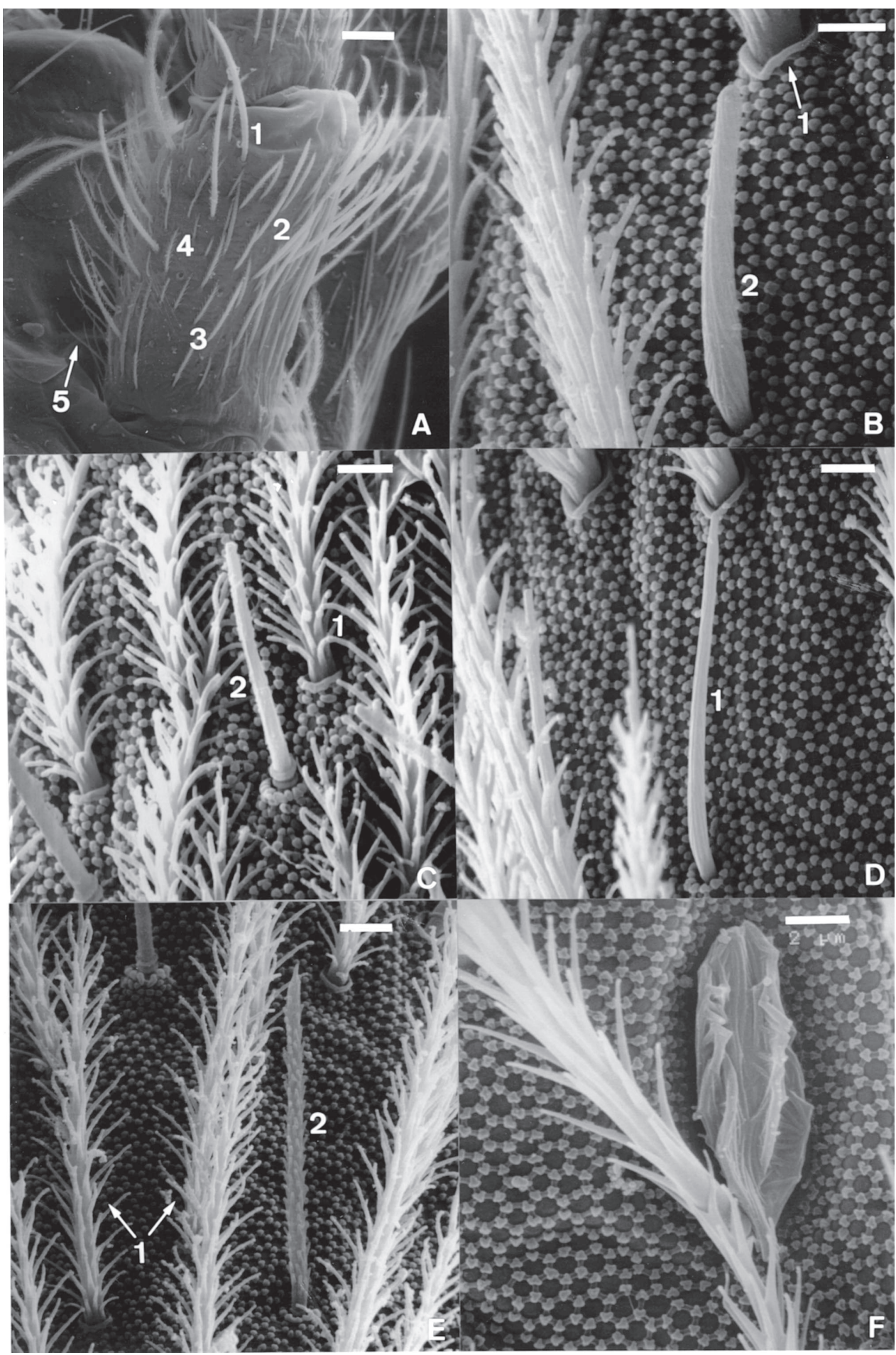
foliácea. Barra $=2 \mu \mathrm{m}$

Fig. 2.- Seta of antenal segment of P. riojana n. sp. A: Antennal segment I where are been indicated the different kind of setae. Bar $=20 \mu \mathrm{m}$. B: 1, macrosetae alveoli; 2, sensorial setae $\mathbf{s}$. Bar= $2 \mu \mathrm{m}$. C: 1 , normal mesosetae; 2, sensorial setae as 2.4. Bar=2 $\mu \mathrm{m}$. D: sensorial setae narrow and long as 2.2. Bar $=2 \mu \mathrm{m}$. E: 1 , normal mesosetae; 2 , sensorial spinulated setae as 2.1 . Bar $=2$ $\mu \mathrm{m}$. F: sensorial setae leaf like. $\mathrm{Bar}=2 \mu \mathrm{m}$. 


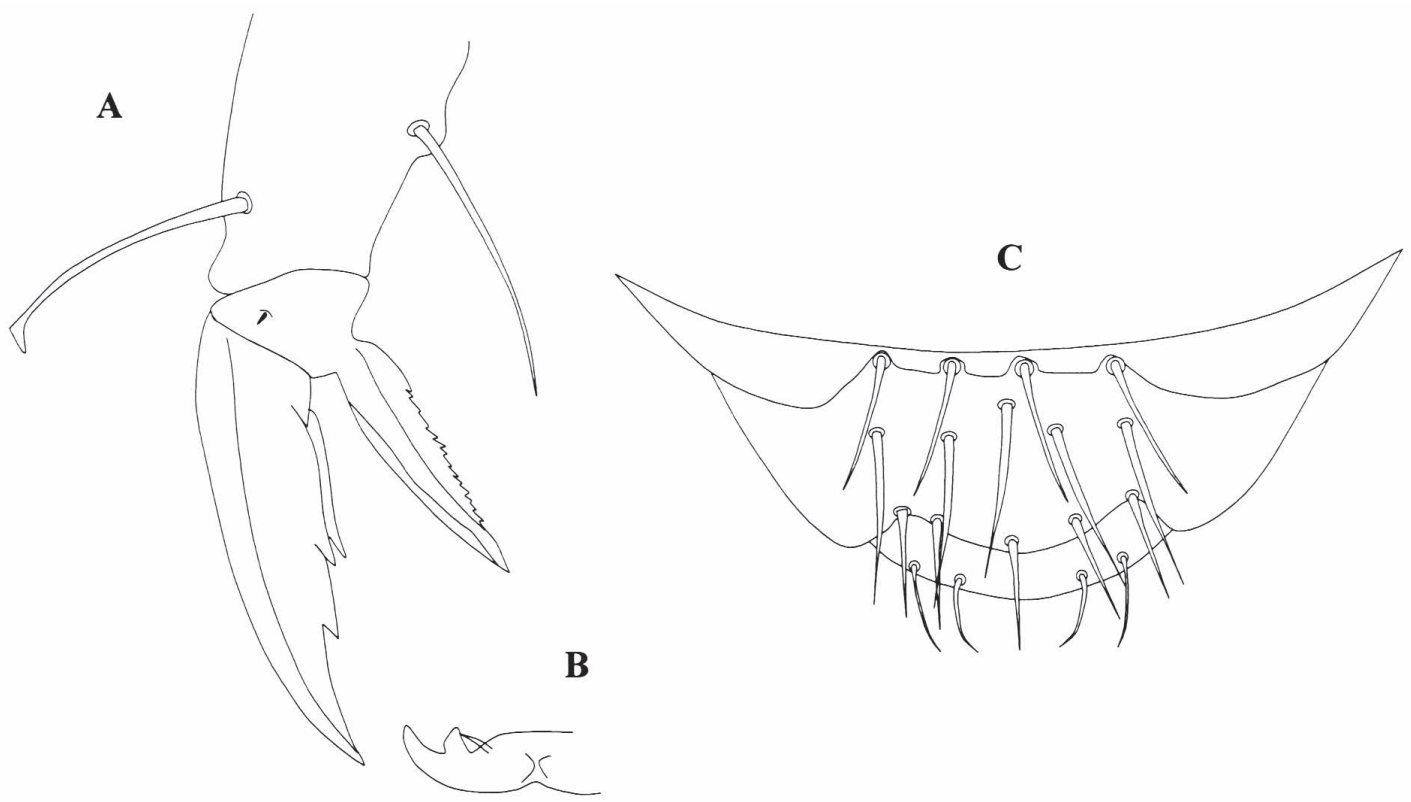

Fig. 3.- P. riojana sp. n. A: uña y empodio; B: mucrón; C: sedas del clípeo y labro.

Fig. 3.- P. riojana n. sp. A: claw and empodium; B: mucro; C: setae of clipeus and labrum.

macrosedas de tamaño medio, con una estriación helicoidal, se encuentran situadas en la parte dorsal del artejo antenal I (Fig. 2, A5); 3): Sedas sensoriales (s) ensanchadas, cortas, más o menos estriadas, y de aspecto foliáceo (Fig. 2, F). Las sedas del tipo 3 son poco numerosas: una en la parte apical externa del artejo antenal II, y las dos sedas $\mathbf{s}$ internas del órgano sensorial del artejo antenal III. Hay una microsensila en la parte apical del artejo antenal IV, fusiforme y característica; no hay vesícula apical exértil en este artejo.

Fórmula clípeo-labral 4/5,5,4 sedas (Fig. 3, C). Las cuatro sedas clipeales son subiguales, largas, finas y lisas. El peine mayor ventrodistal del labro posee de 7 a 9 gruesos dientes, el peine menor lleva un número mayor, aproximadamente de 12 dientes, pero están mucho menos desarrollados. Fórmula de la base del labium: $\mathbf{M}_{\mathbf{1}} \mathbf{m}_{2} \mathbf{r e} \mathbf{l}_{\mathbf{1}} \mathbf{l}_{2}$. La seda $\mathbf{M}_{\mathbf{1}}$ puede aparecer lisa en algún ejemplar, pero siempre asimétricamente, la seda $\mathbf{r}$ es vestigial.

$\mathrm{La}$ fórmula de macrosedas dorsales es R001/0/00/0101+2. Quetotaxia del segmento abdominal II: $\mathbf{a} \mathbf{B} \mathbf{q}_{1} \mathbf{q}_{2}$. Seda $\mathbf{s}$ presente cerca del tricobotrio anterior del segmento abdominal IV.

Uña con la placa dentaria ocupando entre el 65 y el $70 \%$ basal del borde interno. Diente impar bien

Tabla 2.- Comparación de las especies próximas a P. riojana sp. n.

Table 2.-Comparison of nearest species of $P$. riojana $\mathrm{n}$. sp.

\begin{tabular}{|c|c|c|c|c|c|c|c|}
\hline Especie & Autor & Ojos & Espolón patas & Macrosedas dorsales & Seg. Abd. II & Quetotaxia labio & Distribución \\
\hline P. riojana & sp. n. & 2 & Espatulado & $\mathrm{R} 001 / 000 / 0101+2 / \mathrm{s}$ & $-a B q q$ & Mmrell & España: Rioja \\
\hline P. yuca & Christiansen, 1982 & $2-3$ & Espatulado & $\mathrm{R} 001 / ? 00 / 0101+2 / \mathrm{s}$ & paBqq & MMrELL* & México \\
\hline P. mauli & Stomp, 1972 & 5 & Espatulado & $\mathrm{R} 001 / ? 00 / 0101+2 / \mathrm{s}$ & paBqq & MMrELL & Luxemburgo \\
\hline P. terricola & Gisin, 1967 & 6 & Espatulado & $\mathrm{R} 001 / ? 00 / 0101+2 / \mathrm{s}$ & paBqq & MMrELL & $\begin{array}{l}\text { Suiza, Francia y } \\
\text { Luxemburgo }\end{array}$ \\
\hline P. kwartirnikovi & Gama, 1973 & 6 & Puntiagudo & $\mathrm{R} 001 / ? 00 / 0101+2 / \mathrm{s}$ & paBqq & MMRELL & Grecia \\
\hline
\end{tabular}

B: macroseda. M: ciliadas, m: lisas. * ELL son microsedas ciliadas en $P$. yuca 


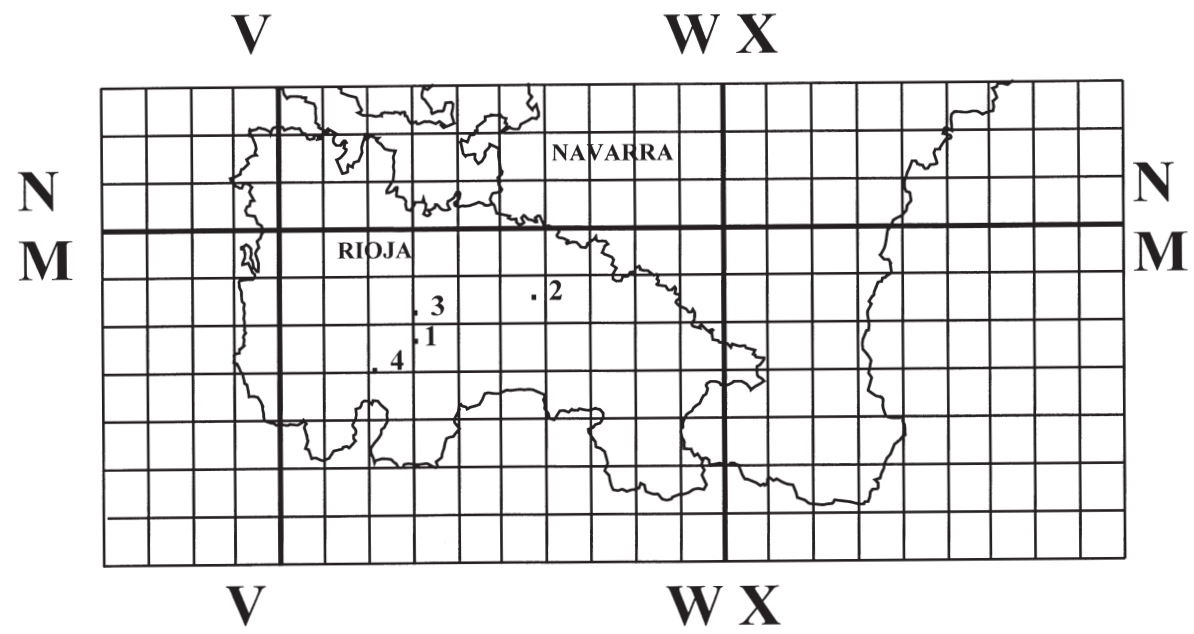

Fig. 4.- Localización geográfica en coordenadas U.T.M. de las cuevas donde ha aparecido P. riojana sp. n.

Fig. 4.- U.T.M. co-ordinates localisation of caves where P. riojana n. sp. has been found.

desarrollado, dientes basales no muy desarrollados, el posterior dos veces más grande que el anterior, que es aproximadamente del tamaño del diente impar distal (Fig. 3, A). Apéndice empodial lanceolado, las aletas llegan hasta el ápice, el borde externo aserrado, con numerosos dientes. Espolón

Tabla 3.- Comparación con otras especies que presentan $2+2$ corneolas.

Table 3.- Comparison of nearest species with $2+2$ eyes.

\begin{tabular}{|c|c|c|c|c|c|c|}
\hline Especie & Autor & ojos & Seda tibiotarsal. & Fórmula Quetotaxica & Abd. II & Labio \\
\hline P. gisini & Christiansen, 1961 & 2 & espatulado & Roo-/00/0100+2/- & paBqq & MmRell \\
\hline P. hirsuta & Delamare, 1949 & 2 & puntiagudo & Roo-/00/0100+4/- & $-a B-q$ & mmrell \\
\hline P. bona & Christiansen \& Bellinger, 1996 & 2 & puntiagudo & $\mathrm{R} 000 / 00 / 0100+2 /-$ & paBqq & MMrELL \\
\hline P. fonsa & Christiansen \& Bellinger, 1996 & 2 & espatulado & $\mathrm{R} 000 / 00 / 0100+2 /-$ & paBqq & MMRELL \\
\hline P. aera & Christiansen \& Bellinger, 1980 & 2 & espatulado & $\mathrm{R} 000 / 00 / 0100+2 /-$ & paBqq & MMrELL \\
\hline P. aera & Christiansen \& Bellinger, 1980 & 2 & espatulado & $\mathrm{R} 000 / 00 / 0100+2 /-$ & paBqq & MMreLL \\
\hline P. thibaudi & Stomp, 1977 & 2 & puntiagudo & $\mathrm{R} 000 / 00 / 0101+2 / 0$ & $-a B q q$ & MmRelL \\
\hline P. reddelli & Christiansen, 1973 & 2 & espatulado & $\mathrm{R} 000 / 00 / 0101+2 / \mathrm{s}$ & paBqq & mmrell \\
\hline P. hercynica & Rusek, 1979 & 2 & espatulado & $\mathrm{R} 001 / 00 / 0100+2 / 0$ & paBqq & MMrELL \\
\hline P. palaciosi & Christiansen \& Reddell, 1986 & 2 & puntiagudo & $\mathrm{R} 001 / 00 / 0100+2 / \mathrm{s}$ & $---q q$ & $-M r E L L$ \\
\hline P. riojana & sp. n. & 2 & espatulado & $\mathrm{R} 001 / 00 / 0101+2 / \mathrm{s}$ & $-a B q q$ & Mmrell \\
\hline P. yuca & Christiansen, 1982 & 2 & espatulado & $\mathrm{R} 001 / 00 / 0101+2 / \mathrm{s}$ & paBqq & MMrELL \\
\hline P. voylesi & Christiansen, 1982 & 2 & puntiagudo & $\mathrm{R} 001 / 00 / 0201+2 / \mathrm{s}$ & -ABqq & mmrell \\
\hline P. bohemica & Rusek, 1979 & 2 & espatulado & $\mathrm{R} 001 / 10 / 0101+2 / 0$ & paBqq & MMrELL \\
\hline$P$ pallida & Gruia, 1977 & 2 & espatulado & $\mathrm{R} 001 / 10 / 0101+2 / 0$ & paBqq & MMrELL \\
\hline P. absoloni & Rusek, 1979 & 2 & puntiagudo & $\mathrm{R} 001 / 10 / 0201+2 / \mathrm{s}$ & pABqq & MMrELL \\
\hline P. alba & Packard, 1873 & 2 & espatulado & $\mathrm{R} 010 / 10 / 0101+2 / 0$ & paBqq & MMrEIL \\
\hline P. alba & Packard, 1873 & 2 & espatulado & $\mathrm{R} 010 / 10 / 0101+2 / 0$ & paBqq & MMrELL \\
\hline P. lleidensis & da Gama, 1984 & 2 & & $\mathrm{R} 011 / 00 / 0101+2 / \mathrm{s}$ & paB-q & \\
\hline P. insularum & Dallai, 1969 & 2 & espatulado & $\mathrm{R} 011 / 10 / 0100+2 / 0$ & -aBqq & \\
\hline P. españoli & Simon \& Selga, 1977 & 2 & espatulado & $\mathrm{R} 011 / 10 / 0101+2 / 0$ & $-a B q q$ & MMrELL \\
\hline P. paprivata & Ellis, 1976 & 2 & espatulado & $\mathrm{R} 011 / 10 / 0101+2 / 0$ & $--\mathrm{Bqq}$ & MMRELL \\
\hline P. aidamar & Luciañez \& Simon, 1994 & 2 & espatulado & $\mathrm{R} 011 / 10 / 0101+2 / \mathrm{s}$ & $-a B q q$ & MMrELL \\
\hline P. cobosae & Luciañez \& Simon, 1994 & 2 & espatulado & $\mathrm{R} 011 / 10 / 0101+2 / \mathrm{s}$ & -aBqq & MMrELL \\
\hline P. lecrerci & Stomp, Massoud \& Thibaud, 1982 & 2 & & $\mathrm{R} 011 / 10 / 0101+2 / \mathrm{s}$ & pABqq & mmrell \\
\hline P. alba & Packard, 1873 & 2 & espatulado & $\mathrm{R} 011 / 10 / 0201+2 / 0$ & $\mathrm{pABqq}$ & MMrELL \\
\hline P. selgae & Simon, 1978 & 2 & espatulado & $\mathrm{R} 011 / 11 / 0201+2 / \mathrm{s}$ & $-a B Q q$ & MMrELL \\
\hline$P$ c ciliata & Ellis, 1974 & 2 & espatulado & $\mathrm{R} 101 / 00 / 0301+2 / \mathrm{s}$ & pABQq & -MrELL \\
\hline P. seyleri & Stomp \& Tommasi-Ursone, 1989 & 2 & espatulado & $\mathrm{R} 111 / 10 / 0201+2 /-$ & pABqq & MmrelL \\
\hline P. baghdadica & Rusek, 1981 & 2 & espatulado & $\mathrm{R} 111 / 10 / 0301+2 / 0$ & $\mathrm{pABQq}$ & -MrELL \\
\hline P. imparipunctata & Gisin, 1953 & 2 & espatulado & $\mathrm{R} 111 / 10 / 0301+2 / 0$ & pABQq & -MrELL \\
\hline
\end{tabular}


tibiotarsal dorsal grueso, espatulado y aproximadamente del tamaño de la seda lisa ventrodistal del tibiotarso III. Patas sin escamas. Tubo ventral con $13+13$ sedas latero-distales, $4+4$ lisas, y 9+9 ciliadas; $13+13$ sedas anteriores, ciliadas; el número y tipo de sedas posteriores no ha podido ser determinado.

Retináculo con $4+4$ dientes y una seda ciliada. Placas dorsales del manubrio con tres o cuatro sedas internas y de siete a diez externas con relación a los dos pseudoporos distales de cada una de ellas. Mucrón corto, el diente distal casi del mismo tamaño que el basal (Fig. 3, B).

DisCusión. Por la fórmula de quetotaxia dorsal y la presencia de seda $\mathbf{s}$ en el complejo tricobotrial anterior del segmento abdominal IV, esta especie se asemeja a $P$. yuca Christiansen, 1982, $P$. mauli Stomp, 1972, P. kwartirnikovi Gama, 1973, y $P$. terricola Gisin, 1967. Se diferencia de todas ellas por no presentar seda $\mathbf{p}$ en del segmento abdominal II y por tener las sedas labiales $\mathbf{m}_{2}, \mathbf{e}, \mathbf{l}_{1}$ y $\mathbf{l}_{2}$ lisas. De las tres últimas, además, se diferencia por el número de ojos. Además de $P$. kwartirnikovi Gama, 1973, se diferencia ya que esta especie tiene $\mathbf{R}$ ciliado en las sedas labiales, y por tanto pertenece a otra línea genealógica (Gama 1984) (Tabla 2). Se realiza la comparación con otras especies con $2+2$ corneolas en la tabla 3.

FAUNA ACOMPAÑANTE. En Cueva Lóbrega: Acherontiella cf. cassagnaui Thibaud, 1967. En Sima del Maestro: Onychiurus argus Denis, 1924. No hay constancia de ninguna otra cueva con fauna conocida en la región estudiada.

DERIVATIO NOMINIS. El nombre de esta especie deriva del de la Comunidad Autónoma dónde se ha encontrado.

\section{AGRADECIMIENTOS}

Agradecemos la colaboración de J. Armendáriz, del Hogar Catalán de la Rioja, por el material facilitado y la del Dr. E. Baquero en la preparación del ejemplar para SEM. Este trabajo forma parte del proyecto de FAUNA IBERICA del Museo Nacional de Ciencias Naturales, CSIC.

\section{Referencias}

Christiansen, K., 1982. Notes on mexican cave Pseudosinella (Collembola: Entomobryidae) with the description of six new species. Folia Entomológica Mexicana, 53: 3-25

Deharveng, L., 1988. Collemboles cavernicoles VII.Pseudosinella bessoni $\mathrm{n}$. sp. et note sur l'évolution morphologique de la griffe chez les Pseudosinella. Revue suisse de Zoologie, 95(1): 203-208.

Deharveng, L. \& Gouze A., 1986. Collemboles cavernicoles. V Une nouvelle Pseudosinella du gouffre de la Pierre-Saint-Martin (France: Pyrénées-Atlantiques). Revue suisse de Zoologie, 93(4): 215-218.

GAMA M.M., 1973. Systématique évolutive des Pseudosinella. IX (Insecta: Collembola). Revue suisse de Zoologie, 80(1): 45-63.

Gama M.M., 1984. Phylogénie des espèces européennes de Pseudosinella (Collembola: Entomobryidae). Annales de la Société Royale Zoologique de Belgique. (Bruxelles), 114(1): 59-70.

Stomp, N., 1972. Deux nouvelles espèces de Pseudosinella endogés d'Europe (Collemboles, Entomobryides). Revue suisse de Zoologie, 79(1): 279-286. 